\title{
Interference Management in Heterogeneous Wireless Networks Based on Context Information
}

\author{
Adrian Kliks \\ Poznan University of Technology, Poznań, Poland \\ akliks@et.put.poznan.pl
}

Andreas Zalonis and Nikos Dimitriou

IASA, National Kapodistrian University of Athens, Greece

\{azalonis I nikodim\}@ phys.uoa.gr

\begin{abstract}
Femtocells and WiFi have been considered as viable concepts in cellular networks to increase overall system capacity. Critical functionalities for the successful integration of such technologies in macro-cellular topologies are related to radio resource allocation and interference management techniques that must ensure seamless intra-cell and inter-cell interoperation among macro- and femto users, as well as between cellular and WiFi users. In this paper, various interference management approaches using rich context information are proposed and discussed for various scenarios of interest, along with a specific assessment for co-channel access points deployment in cellular networks
\end{abstract}

\section{INTRODUCTION}

The need to increase system capacity in wireless networks has driven the research in the field over the past years. As stated in, e.g. [1], [2], the most reliable way to achieve this goal is to reduce the distance between the transmitter and the receiver, thus achieving higher quality links and more spatial radio frequency reuse. Cellular operators enhance their infrastructure with the addition of relays, microcells, and lately with femtocells. Femtocells are low power radio Access Points (APs), providing wireless services to users primarily in home or small-office environments. By assumption, femtocells are deployed by end-users and thus, their locations are dynamic and random. In such a case the Interference Management (IM) deals with proper resource allocation between femtocells (one tier of the network) and macrocells (second tier). Moreover, network operators try to find alternative ways for coverage and capacity increase in densely populated countries. One possible option is to offload the part of the traffic to unlicensed spectrum utilizing WiFi technology [3]. However, the interference between WiFi users seems to be crucial factor that reduce the potential capacity improvement. Since WiFi terminals operate on non-licensed bands, the IM problem cannot be treated as it is typically done in the so-called two-tier approach, e.g. for femtocells. The degree of freedom in interference management and avoidance is strictly limited in this case, since the operator does not have any influence on the other WiFi users and other wireless systems operating in the $2.4 \mathrm{GHz}$ band. Similar analysis can be performed for cellular networks (LTE/4G/WiMax), where the full frequency reuse approach has been applied. In such a situation the whole set of the resources assigned to a certain operator can be used in each separated cell. In all cases the problem of effective yet simple interference management as

\author{
Jad Nasreddine and Fanghua Li \\ RWTH Aachen University, Aachen, Germany \\ jad@inets.rwth-aachen.de \\ Youngwook Ko \\ CCSR, University of Surrey, Surrey, UK \\ y.ko@surrey.ac.uk
}

well as of effective resource allocation (RA) among users become crucial. To control interference, one can listed the two main approaches: (i) interference cancellation techniques, where the user subtracts the strongest interferers from the received signal, and (ii) interference avoidance techniques, especially through RA, where the users try to avoid, instead of suppressing, the interference. For both cases rich context information about the current situation of the terminal plays significant role. Efficient interference management will be impossible if the information about the vicinity of the particular user will be not available. However, depending on the approach, different amount and types of information are - from the one hand available, but can be-from the second hand - required.

The paper is organized as follows. First, IM approaches for various scenarios of interests are discussed in section II along with the available context information for each scenario. In section III a generic system model and the feasibility of cochannel allocation with the use of context information is presented. Novel power control algorithm that takes into consideration and uses the available context information is assessed in section IV. A test case for orthogonal channel assignment is provided in Section V. The paper is concluded in Section VI.

\section{CONTEXT-AWARE INTERFERENCE MANAGEMENT}

Before going into the detailed description of the IM strategies in the aforementioned scenarios, it is worth analyzing shortly the notion context information. Based on [4] context information is the information possessed by the entity that can be used to describe and characterize the current situation of this entity (element of the network). The accuracy of the characterization of the current situation depends not only on the amount of information gathered by the entity but also on the quality of the context information. Moreover, depending on the entity type (e.g. mobile or fixed terminal) the useful and available context information change significantly. For instance some information accessible in cellular networks cannot be obtained in WiFi systems. In the following we address the context information for three different scenarios and try to assess the impact on the system performance.

A. Cellular Networks with Full Frequency Reuse IM in cellular networks relies on some feedback parameters 
sent by user equipment to Base Stations (BSs). Usually these parameters are related to measurements of the received signal strength or the interference plus noise ratio (SINR), on specific frequencies that may assist in determining the propagation losses and its variations. Apart from assessing the link quality between the BS and the mobile, it is important to assess the interference experienced due to activities in other co-channel links, and to estimate the expected consequences to other users after allocating a specific resource to the user of interest.

The traditional resource management techniques in legacy systems have the following characteristics: (i) the cellular infrastructure is considered as fixed and the amount of resources allocated in each cell does not change in short term; (ii) users transfer only channel and signal quality metrics and not any location information; (iii) users do not sense explicitly other systems that may be existing in other frequency bands. One should note that the specifications for LTE-Advanced are considering the use of more rich information especially for the Minimization of Drive-Tests (MDT) procedure [5]; (iv) the baseline configuration of cellular systems does not involve the cooperation between base stations for optimized resource usage; (v) there is no provision for opportunistic resource access or for primary/secondary prioritization of users in RA.

In the case of a macro-cellular architecture with nearly full frequency reuse in adjacent cells, there is a need for optimum RA and IM. The resulting co-channel interference that may be generated needs to be carefully controlled to ensure seamless operation of both cellular and hot-spot area systems. This necessitates the co-ordination among BSs and hot-spot APs to manage the use of the common resources. This co-ordination can be assisted by using rich context information: (i) BS locations - this requires a low update rate and will serve for making long-term network planning decisions; (ii) information about network infrastructure, such as BS power control capabilities; (iii) real-time or almost real-time measurements conducted by users, along with their locations. Each user may collect information regarding its surrounding radio environment, containing even more dynamically updated measurement information; and (v) user requirements on Quality-ofService (QoS).

\section{B. Femtocells in 3G/4G two-tier networks}

Similar approach can be applied in the situation where the use of femtocells is considered. The deployment of femtocells can be seen as an introduction of an additional layer of wireless coverage. However, due to its potential dense deployment, it can produce high level of interference to the macrocell layer. Notice the fact that femto access points (FAPs) can be deployed at any location within a macrocell at any time; they can also be moved from one location to another, therefore RA and IM techniques have to manage the radio resources dynamically to adapt to the rapid variations of the radio environment. Rich context information that can be used by future RA and IM techniques should be defined and organized. This categorization will enable the resource controller to access the required information without delay and store data without flooding the network with unnecessary signaling.
The first rule to consider for collecting and storing context information is the update period of each type of information. Information with high dynamics (e.g. real time path loss, instantaneous power distribution, and interference and SINR patterns) will be stored as close as possible to the terminals (in a terminal or femtocell database). Information with less dynamics (e.g. the characterization of the building and their maps, the position of the femtocells, the propagation and traffic pattern models, user density, and traffic distribution) can be stored also in more distant database such as femtocell database or covering macrocell. Moreover applications using this type of information can download them once the node is turned on and does not need to contact the database all time. Furthermore, the data that need high computation power should be assessed and stored near to network elements with high computational power such as macrocell BSs. For instance interference maps can be built by each macro cell BS using the information collected from the femtocells and users in its coverage.

The data can be categorized based on the equipment responsible of collecting the information. Information, such as the SINR, propagation loss, interference values at a given place and time can be collected by either terminals or FAPs depending on the direction of the link. Other information, such as cell capacity, coverage area can be collected by FAPs.

\section{WiFi operator network scenario}

The frequency bands dedicated for WiFi networks are split into smaller overlapping channels. Within one channel the interference is avoided by the application of the CSMA/CA (Carrier Sense Multiple Access with Collision Avoidance) scheme, in which only one transmitter can be active in the certain time. However, the increase of the number of WiFi users and WiFi networks in a given area can significantly decrease the overall throughput due to more frequent collisions when two or more users start transmitting in the same time. Moreover if the neighboring networks are working in adjacent, and thus overlapping channels (e.g. channel 3 and 4 in ISM band), the signals transmitted in these bands interfere between each other and lead to a reduction in the SINR values. In order to avoid such situations the WiFi network operators should use non-interfering channels (i.e. 1, 6 and 13 in industrial, scientific and medical (ISM) frequency bands). However, since WiFi networks can be installed and implemented locally at homes, total avoidance of interference is not possible. From this point of view coordination between various WiFi APs will be beneficial. In order to better use the common resources such coordination can be also assisted by context information exchange and management. APs can exchange local information about their locations (coordinates), occupied channels with some parameters describing traffic density as well as average delays. In such a case neighboring APs are able to select the best configuration of the occupied channels maximizing the throughput or minimizing the interference.

\section{ASSESSMENT SCENARIO AND MODELING}

In all of the abovementioned scenarios the important issue is to determine the possibility of re-using the same frequency channels for different users and the corresponding consequences related to the generated co-channel interference. Let analyze 
the following system model presented in Fig. 1, where three omnidirectional transmitting APs, named $A, B$, and $C$, and three receiving Mobile Stations (MSs), named 1,2,3, communicate within the same area.

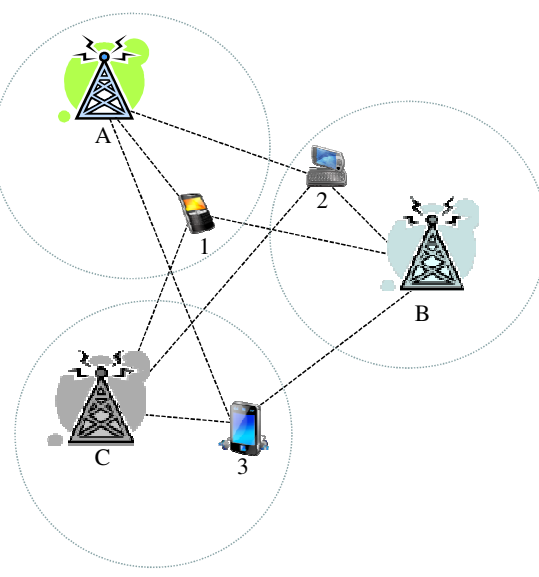

Figure 1. Co-channel allocation scenario

Our objective is to examine how the available context information can help in increasing the efficiency of IM techniques. The analysis is valid for any scenario involving cochannel frequency allocation in neighboring cells; the assumption of 3 co-existing co-channel users within the same area is valid and covers most practical system layout scenarios for macro-cells, femtocells and opportunistic access systems. One such example is cellular OFDMA full frequency reuse scenarios, involving the wide re-use of the same sub-channels. Another example is co-channel LTE femtocells deployment in a specific area, or a WiFi deployment with a provision of re-use of the same sub-channels.

The calculation of the interference in each possible interference scenario of Fig. 1 is based on the parameters in Table I. The total channel gain $g_{m, n}=L(\alpha, d, S, H)$ can be segregated into path-loss component at distance $d$ with attenuation factor $\alpha$, shadowing $S$, and fast fading term $h$. Depending on the scenario of interest and the available context information the exact values or probabilistic models can be used to represent the above parameters. The exact values, in the generic case, are dependent on the positions of the transmitter and the receiver. In each scenario the available channel knowledge can be represented accordingly as a special case of the complete total channel gain $g_{m, n}$. Below an exemplary sum-utility optimization problem is presented as an example, with the intention to be as generic as possible to cover as much as possible sub-problems in the area. Clearly, other classes of generic optimization problems can be also identified. In the ideal case, where we have the exact channel knowledge of all the interconnections in the network and a centralized controller that will collect in real-

TABLE I. EXEMPLARY SET OF CONTEXT PARAMETERS.

\begin{tabular}{|c|l|}
\hline Parameter & \multicolumn{1}{c|}{ Description } \\
\hline $\mathrm{MS}_{m}, m=1,2,3$ & $m$-th MS user (total number of MS is equal to $M$ ) \\
\hline $\mathrm{AP}_{n}, n \in\{A, B, C\}$ & $n$-th AP (total number of AP is equal to $N$ ) \\
\hline$g_{m, n}$ & Total channel gain between the nth AP and mth MS \\
\hline
\end{tabular}

time this information, a generic optimization function that targets to the maximization of a specific function $X($.$) can be for-$ mulated as:

$$
\max \sum_{(m, n)} X_{m, n}\left(\hat{r}_{m, n}, \hat{p}_{m, n}\right)
$$

where the sum is over the tuples $(m, n)$. Power and rate allocated for a $\mathrm{MS}_{m}$ are represented as $p_{m, n}$ and $r_{m, n}$, respectively. Function $X($.$) can be the capacity, the sum goodput, or some-$ thing else. Various optimization functions could be selected depending on the scenario of interest. This generic optimization example can be used as a reference for various practical subproblems in the area of IM and RA in femtocell networks.

\section{Conditions for feasible co-channel allocation}

Each user in Fig. 1 is assumed to have a specific QoS requirement in terms of SINR value, i.e. the $m$-th mobile station $\mathrm{MS}_{m}$ has an SINR requirement equal to $\gamma_{m}$. By considering the conditions that have to be fulfilled by user $\mathrm{MS}_{i}$ to be connected to $\mathrm{AP}_{X}$, it can be shown that there can be feasible power allocation strategy for all three users if the expression concerning the Feasible Power Allocation Criterion (FPAC) is valid:

$$
\mathrm{FPAC}=1-\gamma_{1} \gamma_{2} \gamma_{3} L_{\mathrm{s}}>0
$$

Here $L_{s}$ represents the interference coupling factor that depends on the path losses between the APs and the MSs as has been introduced in [9] for CDMA systems and adopted in this paper for any full-frequency reuse system. This criterion may be integrated with the maximum power conditions that may be imposed by the AP transceiver characteristics.

Herein we assume the knowledge of the location and the QoS requirements of all users, along with the propagation models for all possible user links. It is obvious that, if this information is available in each AP, it can be directly inferred whether there is a feasible co-channel allocation for all users. Thus, the previously described mathematical model can be used to determine feasible power allocation options for each user according to its QoS requirements and location. In case there are more than one connection options using the same frequency (e.g. connection to a macrocell BS or a femtocell AP), this model can be used to identify better connection type.

\section{CONTEXT-AWARE POWER CONTROL}

This section presents the simulation results showing the benefit of exploiting context information for the power allocation of co-channel users in adjacent cells in order to control the generated interference. An area of 3 neighboring omnidirectional cells (each having radius equal to $1 \mathrm{~km}$ ) was simulated. User locations were generated randomly in each cell. The propagation models where assumed to be known. The algorithm for performing power allocation for each user is the following:

1. The user in each cell sends to the AP information regarding the received SINR measurements.

2. The AP calculates the user location (this can be done either by assuming that the user sends directly her/his location information or by assuming that the BS uses some triangulation or other localization determination tech- 
niques, in collaboration with the neighboring BSs that also can locate the user of interest).

3. The AP updated a local database the new user data.

4. The AP sends the updated information for all users to a cluster controller (a cluster Radio Environmental Map REM ).

5. The updated cluster controller is used to calculate the FPAC from expression (2) and to allocate in an optimum fashion the same frequency to users in the adjacent cells. If no feasible solution exists, the users with the higher propagation loss values are allocated different channels to avoid excessive interference.

It was assumed that if the FPAC was positive, any feasible transmitted power vector corresponding to the specific user QoS requirements and user location would be within the power limit bounds of each AP.

This procedure is compared to the case of radio distancebased fractional frequency reuse-based schemes [10] according to which the AP allows full frequency reuse within a radius $R_{c}$ smaller than the cell range (radio distance in this simulation coincides with the user distance from the base station due to the chosen path loss model). The tests were performed assuming a full frequency reuse area of radius $0.5 R_{c}, 0.75 R_{c}$, and $R_{c}$ (denoted as FFR-0.5, FFR-0.75, FFR-1, respectively). The FFR-1 scheme coincided with the case, according to which the AP allocated resources to all users assuming full frequency re-use, and was considered as a lower bound case. In each case, following the co-channel allocation, the SINR of each user was measured. If the SINR $s_{i}$ was below a specified threshold (set to $12 \mathrm{~dB}$ for the tests), the user was considered to be in outage.

Fig. 3 depicts the outage-free frequency reuse utilization (defined as the product of the average channel usage per cell and of the respective average outage rate due to the generated co-channel interference) measured in the 3 neighboring cells. It is apparent that in the case of the uncoordinated resource allocation (FFR-1), users close to the cell borders suffered from excessive intercell interference and thus a considerable outage rate was observed. In the case FFR-0.75, the outage rate is considerably decreased since the distance between co-channel users in different cells is increased and hence the co-channel interference droped. The same trend is observed for the FFR-0.5 scheme that has zero outage rate, which also coincides with the outage rate of the proposed REM-based allocation. However, limiting the full frequency reuse users within a fixed part of each cell may lead to underutilization of resources. The FFR-1 scheme allocates the same channel to all users in each cell, whereas the FFR- 0.75 and FFR- 0.5 schemes exclude around $25 \%$ and $50 \%$ of the users in each cell, respectively, from the common access channel. The proposed scheme, based on calculating the FPAC using REM parameters, outperforms both abovementioned schemes and leads to better utilization of resources, excluding a much lower percentage of users from the common channel allocation and leading to an optimum interference-free frequency channel re-use per user and cell area (see Fig. 3). Its drawback is its dependency on REM context information that might have inaccuracies, which will have a negative impact on power allocation calculations and on frequency channel exploitation.

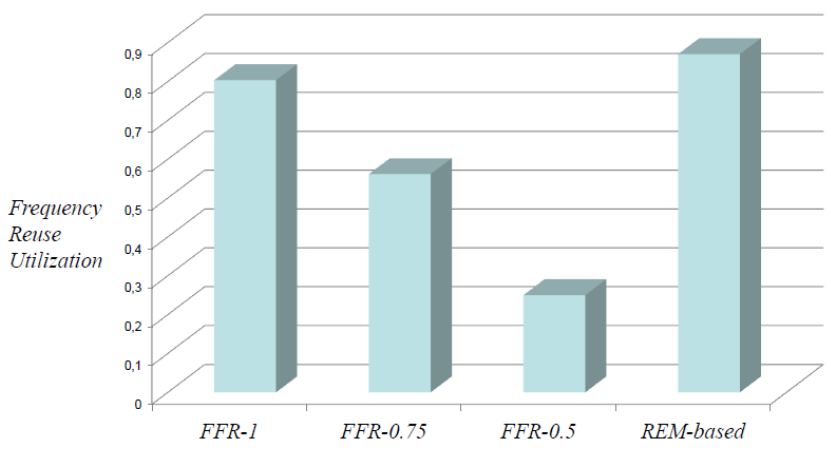

Figure 3. Frequency reuse utilization results.

\section{TEST CASES FOR FEMTOCELLS}

In this scenario, we consider an LTE system where femtoand macro-cell users share a pool of $N_{s c}$ sub-channels (Fig. 4). Co-channel allocation between two tiers of a network can be difficult, especially if a heavily femtocell layer is deployed in one tier. Although co-channel allocation provide high efficiency, the high interference - which is difficult to manage due to femtocell deployment characteristics - consists a challenging problem to deploy such allocation. One solution to this problem is to split the sub-channels using dynamic sub-channel assignment based on traffic distribution into two orthogonal sets, SF and SM, that are used by femto- and macro-cells, respectively. To adapt to the varying system conditions, this assignment is supposed to be performed in long-time scale using, one of the already proposed methods (e.g. [7]). After applying sub-channel assignment, sub-channel allocation to users is performed within each LTE sub-frame (i.e. $1 \mathrm{~ms}$ ) by choosing the sub-channels from a set SF to femtocell users and from a set SM to macrocell users.

Another approach is to use the whole pool of sub-channels for macrocells but with a frequency reuse higher than 1 as in [8]. In this case the femtocells will use the sub-channels allocated to the neighboring macrocells, which are not used by their covering macrocells. This is possible because the interference experienced by the neighboring cells due to femtocell activities is very low, due to the low power of the latter and high propagation losses in indoor-outdoor environments. The main objective of this approach is to maximize the overall capacity of the system and more particularly the capacity of femtocells.

In order to develop an effective method for efficiently identifying a good sub-channel allocation, we use the concept proposed in [8] combined with an opportunistic sub-channel access by femtocells to the channels of the covering macrocell. The same opportunistic access can be also used when orthogonal sub-channels are assigned to femto- and macrocells. In this section we only consider the case of frequency reuse 3 and we consider only the downlink case.

Opportunistic access to macrocell sub-channels by femtocells is possible because not all sub-channels will be used by the macrocell; for instance during the night most of the traffic will be generated from inside the buildings, and therefore most of the macrocell sub-channels will be free. Hence when a femtocell does not find enough channels from those allocated 


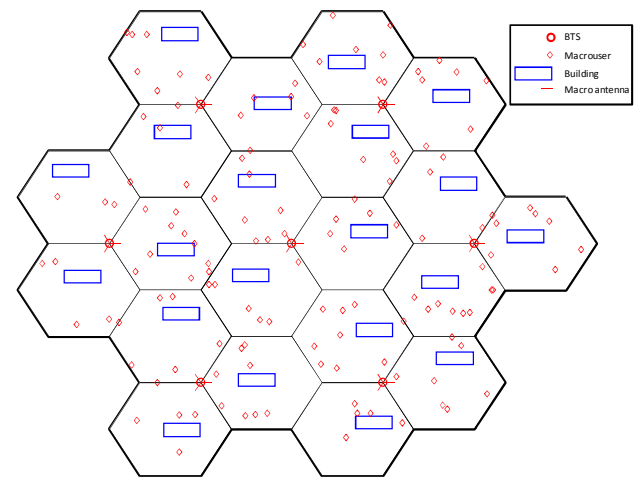

Figure 4. Macrocell layout with 7 base stations (i.e., 21 sectors) and one building block in each sector for urban scenario

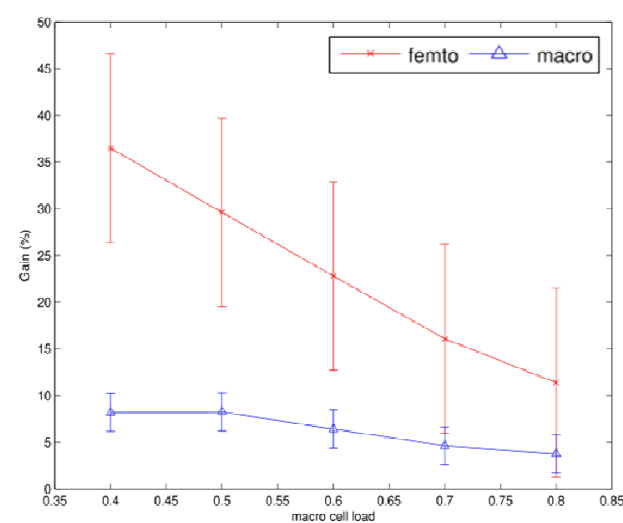

Figure 5. Shannon capacity when using the proposed method (new) or traditional one in urban scenario as a function as network load with a deployment rate of 0.8

to the neighboring cells to serve its users, it will try to opportunistically access candidate macrocell channels. To do this we assume that the femtocell is provided with context information and knows the channel allocation or the allocation map of its covering macrocell. This can be done by either sensing techniques (as proposed in e.g. [11]), or decoding the allocation map sent through macrocell control channels. We also propose that the femtocells of the same building cooperate to perform their sub-channel allocation. This is important when femtocells will be heavily deployed in indoor environments since the interference between these femtocells can be very high due to the small distances that may separate them. To have coordination among them, we assume that the operator will deploy a building-REM (a REM for each building) [12]. This local REM will be a real time shared database enabling femtocells to register and update their sub-channel allocation. By cooperating, the FAPs of the same building, which are deployed in close apartments, can use different sets of sub-channels to avoid high interference. For our simulations we consider an urban scenario where FAPs are located inside buildings with different deployment ratios. Within each building there are 6 floors and 40 rooms of $10 \times 10 \mathrm{~m}^{2}$ in each floor. The FAPs are placed randomly inside the rooms and the femtocell UEs are located within a specified minimum separation distance to the FAP. All macro UEs are assumed to be located outdoors.

In Fig. 5 we present the Shannon average capacity gain of a femtocell and macrocell users in the urban scenario when the proposed method is applied compared to the case where a modified version of the approach proposed in [8] is used. The figure shows that the capacity gain for femtocell users is between $12 \%$, and $37 \%$ for high deployment ratio (i.e. $80 \%$ ). In addition, there is also a gain in the capacity of macro cells, which is around $5 \%$. This gain is due to the fact that the femto users accessing to the unused sub-channels of their covering BSs are very far from the nearest macro BS using the same subchannel. Thus, the generated interference is reduced. Moreover this capacity enhancement is a decreasing function of the cell load since the unoccupied sub-channels allocated to macrocells will decrease and the interference from neighboring macrocells will increase. The results in this section can be seen as an upper bound of the performance of the algorithm if applied to operational system due to the possible lost opportunities.

\section{Conclusions}

The paper discussed resource allocation and interference management techniques for regulating the co-existence of multiple cellular networks, either based on macrocells or on coexisting macrocells/femtocells. The focus was on possible coordination schemes that would exploit the context information that can be gathered by the network nodes (terminals and access points) and on the identification of effective management techniques that can exploit this information (orthogonal or cochannel allocation techniques). The future work will aim at comparing the abovementioned methods in order to identify the related tradeoffs for each and to determine the scenarios for which each approach is more applicable.

\section{ACKNOWLEDGEMENT}

This work has been supported by the ACROPOLIS project (Advanced coexistence technologies for radio optimization in licensed and unlicensed spectrum - contract number 257626).

\section{REFERENCES}

[1] V. Chandrasekhar, J. G. Andrews, "Femtocell Networks: A Survey", IEEE Communication Magazine, Sept. 2008.

[2] M. Mueck et al., "Smart Femto-Cell Controller Based Distributed Cognitive Pilot Channel", CROWNCOM'09, Hannover, Germany, 2009

[3] M. Andrews, V. Capdevielle, A. Feki, and P. Gupta. "Autonomous spectrum sharing for mixed LTE femto and macro cells deployments", in Proc. of INFOCOM WiP, 2010

[4] A.K. Dey. Architectural Support for Building Context-Aware Applications. PhD thesis, Georgia Institute of Technology, December 2000.

[5] 3GPP TS 37.320 - Universal Terrestrial Radio Access (UTRA) and Evolved Universal Terrestrial Radio Access (E-UTRA); Radio measurement collection for Minimization of Drive Tests (MDT); Overall description; Stage 2 (Release 10)

[6] D. Lopez-Perez, et al., "OFDMA Femtocells: A Roadmap on Interference Avoidance”, IEEE Communication Magazine, Sept. 2009.

[7] Q. Su, et al., "A Distributed Dynamic Spectrum Access and Power Allocation Algorithm for Femtocell Networks", WCSP'09), 2009.

[8] T. Kim, T. Lee, "Throughput enhancement of macro and femto networks by frequency reuse and pilot sensing," IPCCC'08, Dec. 2008.

[9] N. Dimitriou, "Network Planning \& Resource Management Issues for Mobile Multimedia CDMA Systems", VTC 2004-Spring, 2004.

[10] J. Jackson, J.Roy, V. Vaidehi, "Analysis of Frequency Reuse and Throughput Enhancement in WiMAX Systems", Wireless Personal Communications, Springer Netherlands, April 2010.

[11] J. Nasreddine, J. Riihijärvi, and P. Mähönen, "Location-based adaptive detection threshold for dynamic spectrum access," DySPAN'10, 2010.

[12] T. Cai, et al., "Design of Layered Radio Environment Maps for RAN Optimization in Heterogeneous LTE Systems," IEEE PIMRC 2011 